\title{
Процессы переноса экситонных возбуждений и релаксация в низкоразмерных полупроводниковых гетероструктурах с квантовыми ямами
}

\author{
() В.Я. Алешкин ${ }^{+*}$, Л.В. Гавриленко ${ }^{+*}$, Д.М. Гапонова ${ }^{+*}$, З.Ф. Красильник ${ }^{+*}$, Д.И. Крыжков \\ + Институт фозики микроструктур Российской академии наук, \\ 603950 Нижний Новгород, Россия \\ * Нижегородский государственный университет им. Н.И. Лобачевского, \\ 603950 Нижний Новгород, Россия \\ E-mail: dmg@ipmras.ru
}

(Получена 9 июня 2016 г. Принята к печати 16 июня 2016 г.)

В гетероструктурах $\mathrm{AlGaAs} / \mathrm{GaAs}$ методом спектроскопии возбуждения фотолюминесценции исследовались процессы, связанные с переносом экситонных возбуждений между туннельно-несвязанными квантовыми ямами и изменением встроенного электрического поля. Наблюдалось изменение интенсивности сигнала низкотемпературной фотолюминесценции (при $4.2 \mathrm{~K}$ ) из более широкой квантовой ямы при совпадении кванта энергии лазера накачки с энергией экситонного перехода в узкой яме. Изменение положения максимума и интенсивности фотолюминисценции из более широкой квантовой ямы при возбуждении вблизи экситонных резонансов в узкой квантовой яме объясняется влиянием квантово-размерного эффекта Штарка на процесс экситонной рекомбинации.

\section{1. Введение}

Возможности управления процессами переноса возбуждений (экситон, электронно-дырочная пара) в полупроводниках во многом определяют эффективность оптических процессов, лежащих в основе работы фотодетекторов, оптических модуляторов, светодиодов и лазеров. В частности, определенный интерес представляет конкуренция процессов переноса возбуждений между отдельными элементами низкоразмерной полупроводниковой структуры (квантовыми ямами, нитями или точками) и процессов излучательной или безызлучательной релаксации возбуждений. Явление переноса энергии между экситонными состояниями в квантовых ямах (КЯ), разделенных широкими туннельно-непрозрачными барьерами, описано в ряде экспериментальных работ см., например, [1-3]. Механизмы, предлагаемые для описания данного явления, различаются, однако чаще всего основываются на так называемой модели Фёрстера-Декстера [4-6]. Модель подразумевает безызлучательное электростатическое взаимодействие между диполями, и это, безусловно, справедливо, например, для взаимодействия возбужденных состояний примесей в кристаллах. В полупроводниковых структурах в равновесных условиях нет статических диполей. В неравновесных условиях поляритоны и экситоны в процессе излучения света представляют собой осциллирующие диполи, поэтому электростатическое описание неприменимо для процесса передачи энергии. Мы связываем изменение интенсивности излучения КЯ при резонансном и нерезонансном возбуждении именно с излучательным переносом экситонных возбуждений. Однако, как будет показано далее, для детального количественного описания изменений интенсивности фотолюминесценции (ФЛ) кроме эффекта ближнепольного переноса экситон- ных возбуждений следует учесть влияние встроенного в структуру электрического поля $[7,8]$.

\section{2. Методика эксперимента}

Исследованные гетероструктуры $\mathrm{GaAs} / \mathrm{Al}_{x} \mathrm{Ga}_{1-x} \mathrm{As}$ $(x=0.45)$ были выращены методом газофазной эпитаксии из металлоорганических соединений (МОГФЭ) на полуизолирующих подложках GaAs и содержали 2 КЯ различной ширины $(10-20$ нм), разделенные барьерами шириной 15 нм. Такая ширина барьеров позволяет считать их туннельно-непрозрачными при времени жизни фотовозбужденных носителей в КЯ < 1 нс [9]. Параметры гетероструктур и энергии основных межзонных переходов (без учета энергии связи экситона) приведены в таблице. На рис. 1 схематически изображены зона проводимости и положение первых трех электронных уровней в исследовавшихся гетероструктурах. Энергия отсчитывается от дна зоны проводимости в GaAs.

Параметры гетероструктур

\begin{tabular}{|c|c|c|c|}
\hline $\begin{array}{l}\text { Толщина } \\
\text { слоя, нм }\end{array}$ & Материал & $x$ & $\begin{array}{c}\text { Энергии } \\
\text { переходов, эВ }\end{array}$ \\
\hline 40 & GaAs & & \\
\hline 50 & $\mathrm{Al}_{x} \mathrm{Ga}_{1-x} \mathrm{As}$ & 0.45 & \\
\hline 10 & GaAs (QW1) & & $\begin{array}{cc}e 1 h h 1 & 1.558 \\
e 1 l h 1 & 1.571\end{array}$ \\
\hline 15 & $\mathrm{Al}_{x} \mathrm{Ga}_{1-x} \mathrm{As}$ & 0.45 & \\
\hline 16 & GaAs (QW2) & & $\begin{array}{ll}e 1 h h 1 & 1.537 \\
e 1 l h 1 & 1.554\end{array}$ \\
\hline $\begin{array}{l}20 \\
50\end{array}$ & $\begin{array}{l}\mathrm{Al}_{x} \mathrm{Ga}_{1-x} \mathrm{As} \\
\mathrm{GaAs} \text { (буфер) } \\
\mathrm{GaAs} \text { (подложка) }\end{array}$ & 0.45 & \\
\hline
\end{tabular}




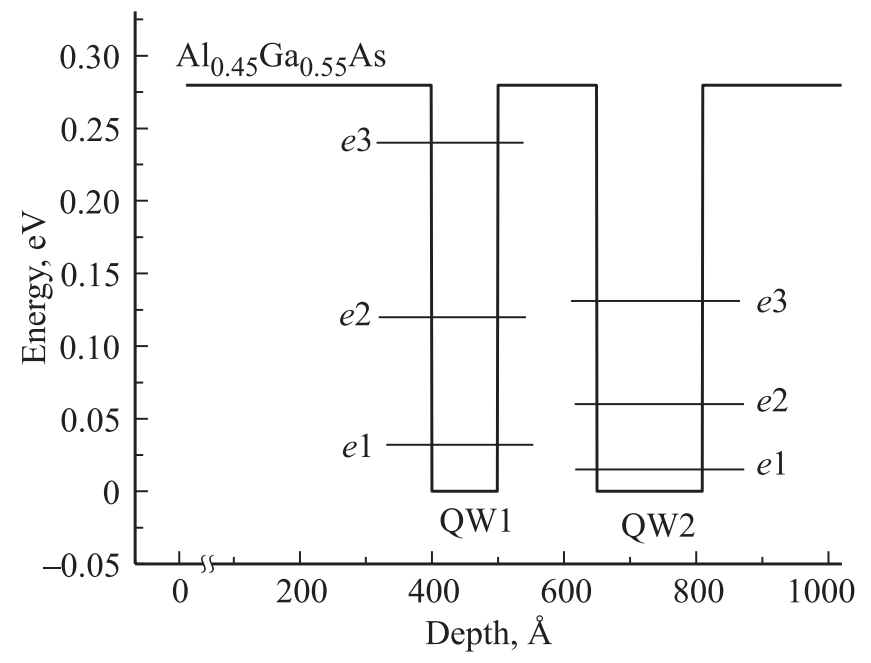

Рис. 1. Схематическое изображение положения дна зоны проводимости в исследовавшейся гетероструктуре. Показаны энергии электронных уровней в КЯ.

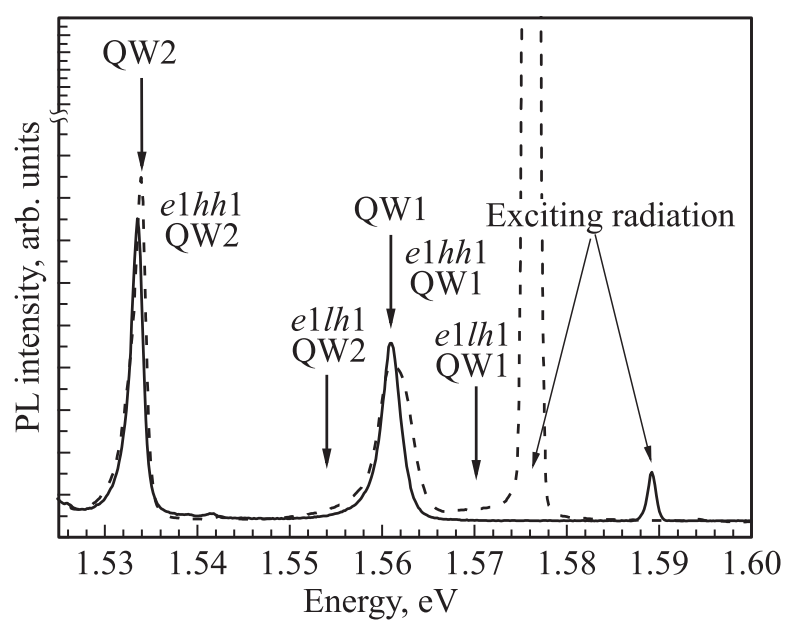

Рис. 2. Спектры фотолюминесценции (PL) гетероструктуры GaAs/AlGaAs $(x=0.45)$ с КЯ 10 нм (QW1) и 16 нм (QW2) в использовавшейся ранее [7] геометрии эксперимента (штриховая кривая) и после модификации экспериментальной установки (сплошная кривая). Мощность возбуждения 0.5 мкВт в обоих случаях.

Экспериментально измеренные спектры низкотемпературной ФЛ и возбуждения ФЛ (ВФЛ) были получены при накачке непрерывным титан-сапфировым лазером „Matisse TR“, перестраиваемым в области 700-950 нм с шагом 0.2 нм. Мощность возбуждения варьировалась от 0.1 мкВт до 50 мВт. Для анализа сигнала ФЛ использовался двойной решеточный спектрометр с разрешением 0.3 нм. Регистрация сигнала осуществлялась с помощью CCD-камеры. Температура измерений составляла $T=4.2$ К. На рис. 2 приведены спектры ФЛ исследовавшейся гетероструктуры, положения экситонных переходов в узкой (QW1) и широкой (QW2) квантовых ямах обозначены стрелками. Спектр ФЛ, полученный в ис- пользовавшейся нами ранее геометрии эксперимента [7], изображен штриховой кривой. В спектре присутствует узкий интенсивный пик с энергией $\sim 1.576$ эВ, возникающий из-за попадания в монохроматор излучения возбуждающего лазера, что делало невозможным измерение интенсивности основного экситонного перехода $e 1 h h 1$ при непосредственном резонансном возбуждении. Поэтому в расчетах предполагалось, что линия основного экситонного резонанса $e 1 h h 1$ имеет ту же ширину, что и линия экситонного перехода с участием легких дырок $e 1 l h 1$, но в 3 раза больше по амплитуде [9].

Для решения этой проблемы схема эксперимента была перестроена таким образом, чтобы отраженный от поверхности образца лазерный луч не попадал в собирающую оптическую систему. При этом основной вклад излучения возбуждающего лазера в регистрируемый сигнал определяется точечными сильно рассеивающими объектами, такими как микроповреждения или загрязнения поверхности образца.

Решетка монохроматора выставлялась в нулевой порядок, и на CCD-матрице получалось изображение поверхности образца. Возбуждающий лазер фокусировался в достаточно малое пятно $(\sim 20$ мкм $)$ и на образце выбирался участок с наименьшим количеством рассеивающих объектов, на котором в дальнейшем проводились измерения. Такая методика позволила на несколько порядков уменьшить вклад излучения возбуждающего лазера в регистрируемый сигнал.

Сплошной кривой на рис. 2 изображен спектр ФЛ, полученный после модификации экспериментальной установки. Пик на длине волны 1.589 эВ по-прежнему соответствует рассеянному излучению возбуждающего лазера, однако его интенсивность меньше интенсивности ФЛ из КЯ.

\section{3. Результаты и обсуждение}

Рассмотрим в рамках модели ближнепольного излучательного переноса, как соотносятся интенсивности излучения в системе из двух КЯ. Интенсивность излучения из широкой КЯ QW2 $\left(I_{\mathrm{QW} 2}\right)$ в зависимости от частоты излучения возбуждающего лазера $\omega$ можно записать в виде

$$
\begin{aligned}
I_{\mathrm{QW} 2}(\omega) \propto & {\left[1-\alpha_{\mathrm{QW} 1}(\omega)\right] \alpha_{\mathrm{QW} 2}(\omega) } \\
& +\alpha_{\mathrm{QW} 1}(\omega)\left(\alpha_{h}+\alpha_{l}+\alpha_{2}^{F}\right) / 2
\end{aligned}
$$

Здесь первое слагаемое отвечает межзонному поглощению излучения возбуждающего лазера в QW2 $\left(\alpha_{\mathrm{QW} 1, \mathrm{QW} 2}-\right.$ коэффициенты поглощения излучения плоской волны в соответствующей КЯ), а второе перепоглощение излучения из первой КЯ второй КЯ. Коэффициенты поглощения ближнепольного излучения, $\alpha_{h}$ и $\alpha_{l}$, зависят от расстояния между КЯ следующим 
образом (подробно описано в работе [9]):

$$
\begin{gathered}
\alpha_{h}=\frac{W_{h}}{I}=\frac{9 \pi}{32}\left(\frac{e^{2}}{\hbar c}\right) \frac{\left|\chi_{h}\right|^{2}}{\varepsilon^{5 / 2} \mu^{3 / 2} k^{4} R^{4}} \theta\left(\hbar \omega-E_{t r}\right), \\
\alpha_{l}=\frac{W_{l}}{I}=\frac{\pi}{4}\left(\frac{e^{2}}{\hbar c}\right) \frac{\theta\left(\hbar \omega-E_{t r}\right)\left|\chi_{l}\right|^{2}}{\varepsilon^{5 / 2} \mu^{3 / 2} k^{4} R^{4}}\left(\frac{15}{8}+\varepsilon \mu R^{2} k^{2}\right),
\end{gathered}
$$

где $W_{h, l}$ - мощность, поглощаемая при переходах из подзон тяжелых, легких дырок, $E_{t r}$ - разность энергий между соответствующими электронным и дырочным уровнями, $I=(2 / 3)\left(d^{2} \omega^{4} / c^{3}\right) \varepsilon^{1 / 2} \mu^{3 / 2}-$ интенсивность излучения диполя (тяжелого экситона в QW1), $\varepsilon$ и $\mu-$ диэлектрическая и магнитная проницаемости, $k=\omega / c$, $R$ - расстояние от диполя до КЯ, $\chi_{h . l}$ - интегралы перекрытия волновых функций электронов и легких (тяжелых) дырок. Коэффициент $\alpha_{2}^{F}$ соответствует поглощению дипольного излучения в плоскости соседней КЯ при падении под скользящими углами, т.е. на значительных расстояниях от диполя (больше боровского радиуса экситона).

Таким образом, интенсивность ФЛ в более широкой яме, $I_{2}(\omega)$, в зависимости от частоты излучения возбуждающего лазера можно записать как

$$
I_{2}(\omega)=I_{2}^{0}(\omega)+\beta(\omega) I_{1}^{0}(\omega),
$$

где $I_{1,2}^{0}(\omega)=\alpha_{\mathrm{QW} 1, \mathrm{QW} 2}(\omega) P-$ интенсивность излучения соответствующей КЯ при отсутствии второй КЯ и при возбуждении плоской волной с мощностью $P$. При этом из формулы (1) коэффициент, определяющий влияние узкой КЯ на ФЛ широкой КЯ будет

$$
\beta(\omega)=\left(\alpha_{h}+\alpha_{l}+\alpha_{2}^{F}\right) / 2-\alpha_{\mathrm{QW} 2}(\omega) .
$$

Таким образом, $\beta(\omega)$ устанавливает однозначное соответствие между величиной резонансного возрастания интенсивности сигнала ФЛ из широкой КЯ и увеличением сигнала экситонной ФЛ узкой КЯ, возникающего при резонансном возбуждении в легкий или тяжелый экситон этой КЯ.

На рис. 3, $a$ приведены спектры ВФЛ узкой (QW1) и широкой (QW2) квантовых ям, а на рис. 3, $b$ - спектры ФЛ перехода $e 1 h h 1$ в QW2 при возбуждении вблизи основного экситонного перехода $e 1 h h 1$ в QW1 при мощности возбуждения 10 мкВт. Стрелками отмечено положение основных переходов в QW1. Собственных резонансов в QW2 с энергиями 1.558 и 1.571 эВ нет, увеличение интенсивности в данных областях может быть связано с ближнепольным переносом излучения из QW1. Пик с максимумом 1.569 эВ в спектре ВФЛ широкой КЯ соответствует переходу $e 1 h h 3$ в этой же КЯ, в спектре ВФЛ узкой КЯ особенностей в данной области энергий возбуждения нет. Как можно видеть из рис. $3, a$, отношение увеличения интенсивности в спектре ВФЛ узкой КЯ для тяжелого, $e 1 h h 1$, и легкого, $e 1 l h 1$, экситонов (энергии 1.56 и 1.58 эВ соответственно) $\sim 3$, в то время как увеличение сигнала из QW2 на этих энергиях различается в 4.5 раза.
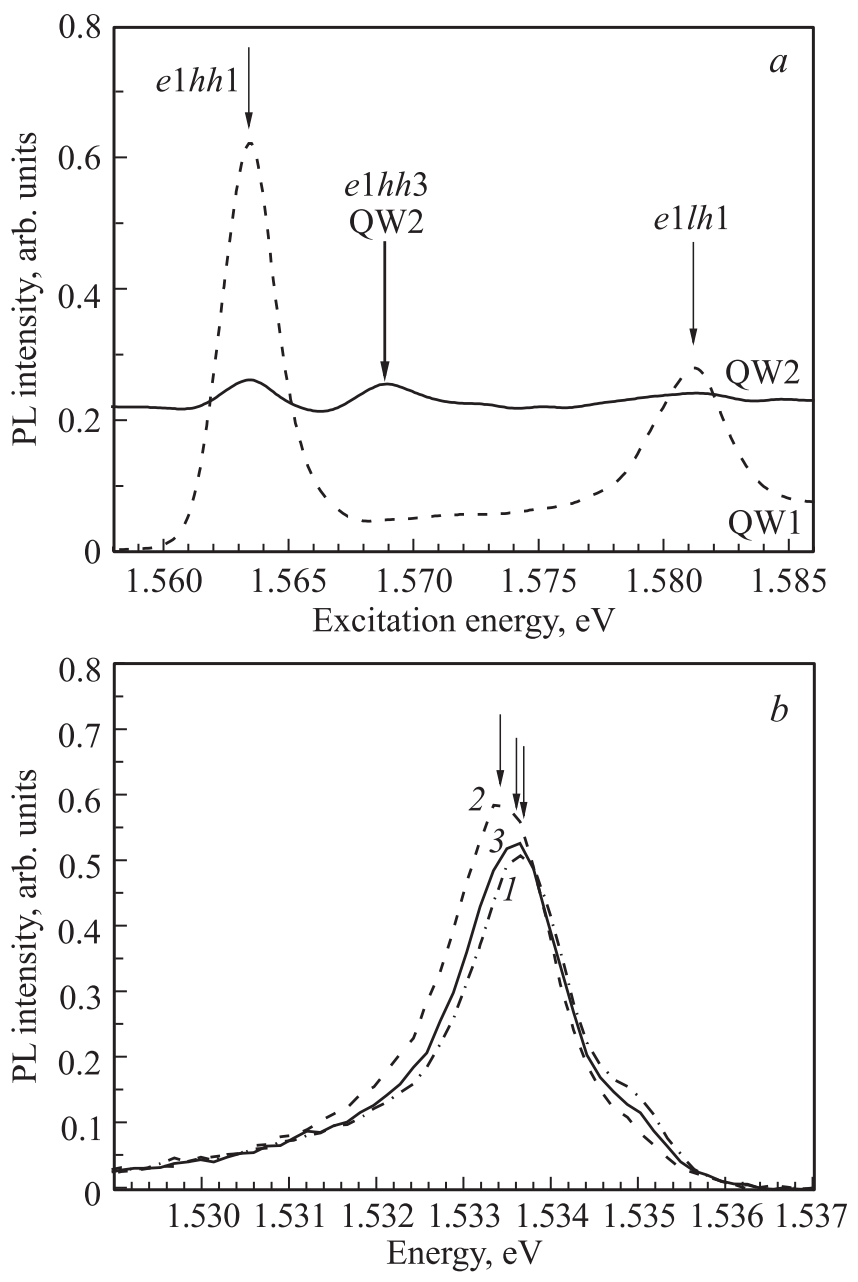

Рис. 3. $a-$ спектры возбуждения фотолюминесценции (PL) гетероструктуры GaAs/AlGaAs $(x=0.45$, ширина QW1 10 нм, ширина QW2 16 нм, ширина барьера между ними 15нм) при мощности возбуждения 10 мкВт; штриховая кривая регистрация при энергии перехода $e 1 h h 1$ в QW1 (1.563 эВ), сплошная кривая - регистрация при энергии перехода $e 1 h h 1$ в QW2 (1.581 эВ); положения возможных переходов указаны вертикальными стрелками. $b-$ изменение положения максимума фотолюминесценции (PL) elhh1 QW2 при возбуждении вблизи основного резонанса в QW1 - при энергиях возбуждения 1.562 (1), 1.563 (2), 1.564 эВ (3).

В спектрах ВФЛ той же гетероструктуры, полученных при мощности возбуждения 200 мкВт (рис. 4, $a$ ), для QW1 отношение увеличения интенсивностей линий, соответствующих поглощению тяжелого и легкого экситонов (1.56 и 1.58 эВ) равно 2.4, тогда как для QW2 соотношение увеличения интенсивностей в спектре ВФЛ на этих длинах волн составляет 1.5 .

В соответствии с выражениями (4) и (5) соотношение увеличения интенсивностей в спектре ВФЛ из QW2 c энергиями, относящимися к поглощению тяжелого и легкого экситонов в QW1 (1.56 и 1.58 эB), пропорционально отношению увеличения интенсивностей линий в спектре ВФЛ из QW1 с соответствующими энергиями. 
Коэффициент пропорциональности $\beta\left(\omega_{e 1 h h 1}\right) / \beta\left(\omega_{e 1 l h 1}\right)$, где $\omega_{e 1 h h 1}$ и $\omega_{e 1 l h 1}$ - частоты, соответствующие тяжелому и легкому экситонам в QW1. Этот коэффициент не зависит от мощности возбуждения, что не соответствует приведенным экспериментальным результатам. Кроме того, теоретические оценки, выполненные для данной структуры в работе [9], показали, что $\beta\left(\omega_{e 1 h h 1}\right) \approx \beta\left(\omega_{e 1 l h 1}\right)$ и соотношение резонансных пиков в спектре ВФЛ QW2 должно быть просто равно соотношению экситонных пиков в спектре ВФЛ QW1, что так же не выполняется.

Приведенные выше экспериментальные результаты говорят о том, что объяснить наблюдаемый эффект только в рамках модели ближнепольного переноса не удается. Какой же иной механизм может приводить к подобному эффекту? На рис. $3, b$ показано изменение положения линии, соответствующей основному экситонному переходу $e 1 h h 1$ в QW2 (в области 1.53 эВ) при изменении длины волны возбуждения вблизи перехода $e 1 h h 1$ в QW1 (1.563 эВ). Штрихпунктирная кривая 1 соответствует возбуждению с энергией 1.562 эВ, т.е. на 1 мэВ меньше энергии тяжелого экситонного перехода в узкой КЯ. Пик ФЛ, соответствующий переходу $e 1 h h 1$ в QW2, расположен при этом на 1.5336 эВ. При резонансном возбуждении узкой КЯ (штриховая кривая 2 на рис. $3, b)$ максимум ФЛ из QW2 наблюдается на 1.5334 эВ, интенсивность ФЛ возрастает. Кривая 3 соответствует энергии возбуждения 1.565 эВ, при этом интенсивность $Ф$ Л QW2 снова уменьшается и смещается практически к своему начальному значению.

Изменение положения линии ФЛ и ее интенсивности может быть обусловлено изменением величины встроенного электрического поля, т. е. квантово-размерным эффектом Штарка. Как было показано в [10], из-за остаточных примесей в барьерах гетероструктур GaAs/AlGaAs, выращенных из металлоорганических соединений, концентрация носителей заряда в КЯ может достигать $10^{14}-10^{15} \mathrm{~cm}^{-2}$. При захвате на долгоживущие состояния они могут создавать встроенное электрическое поле. Из сравнения рис. $3, b$ и $4, b$ видно, что влияние встроенного электрического поля может приводить как к увеличению, так и к уменьшению энергии линии ФЛ, относящейся к широкой КЯ при резонансном возбуждении более узкой КЯ. На рис. $4, b$ сплошная кривая 1 соответствует возбуждению с энергией 1.562 эВ, т.е. на 1 мэВ меньше энергии тяжелого экситона в QW1. При этом максимум линии ФЛ из QW2 расположен на 1.5329 эВ. Штрихпунктирная кривая 2 соответствует резонансному возбуждению перехода $e 1 h h 1$ в узкой КЯ. При этом интенсивность ФЛ из QW2 максимальна, пик расположен на 1.5334 эВ. При возбуждении излучением с энергией 1.565 эВ максимум линии ФЛ из QW2 (штриховая кривая 3 на рис. $4, b$ ) смещается обратно в сторону меньших энергий (1.533 эВ), интенсивность ФЛ падает.

Одно из возможных объяснений приведенных выше спектров состоит в том, что фотовозбужденные электроны и дырки, возникающие при резонансном возбуждении
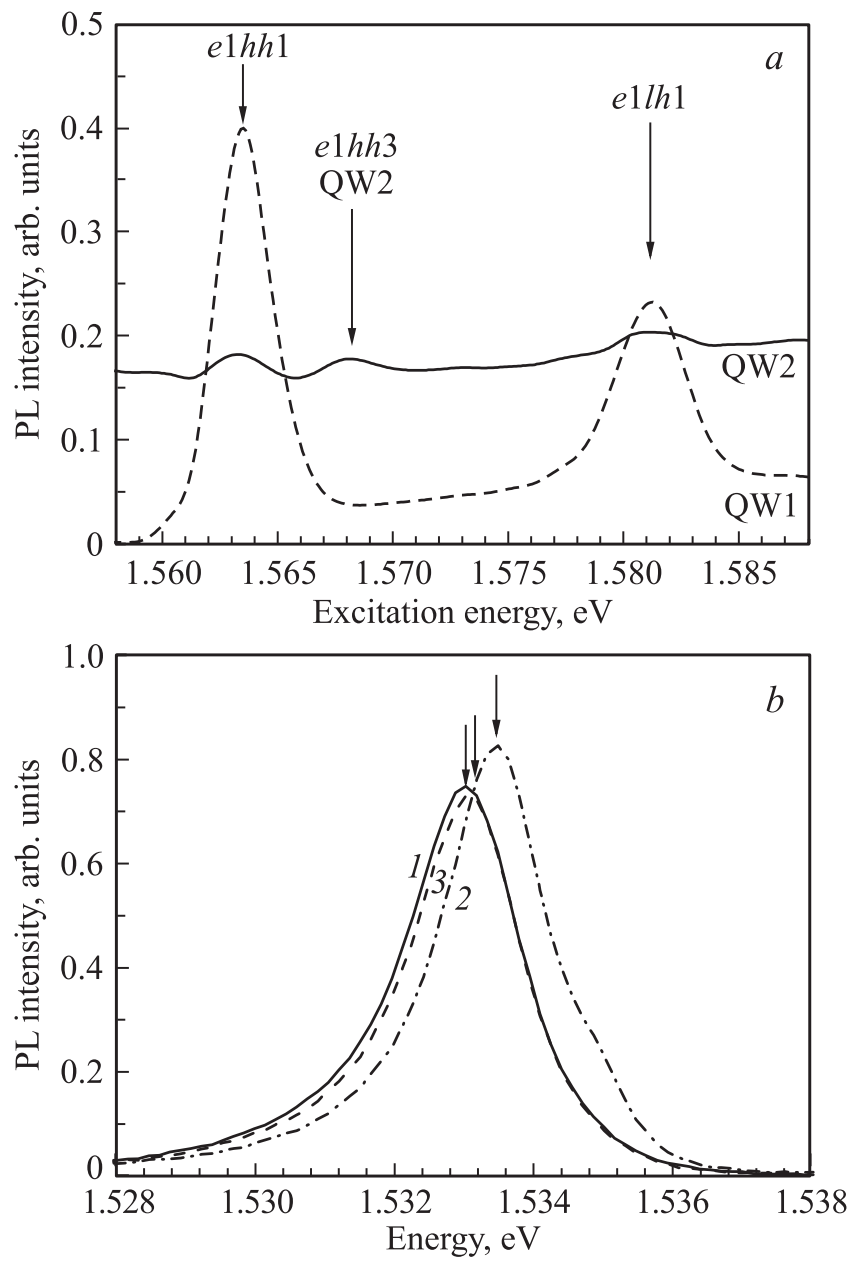

Pис. 4. $a-$ спектры возбуждения фотолюминесценции (PL) гетероструктуры GaAs/AlGaAs $(x=0.45$, ширина QW1 10 нм, ширина QW2 16 нм, ширина барьера между ними 15 нм). Мощность возбуждения 200 мкВт. Штриховая кривая - регистрация при энергии перехода $e 1 h h 1$ в QW1 (1.563 эВ); сплошная кривая - регистрация при энергии перехода $e 1 \mathrm{hh} 1$ в QW2 (1.581 эВ), положения возможных переходов указаны вертикальными стрелками. $b-$ изменение положения фотолюминесценции (PL) e1hh1 QW2 при возбуждении вблизи основного резонанса в QW1 - при энергиях возбуждения 1.562 (1), 1.563 (2), 1.564 эВ (3).

экситонного перехода в QW1, изменяют напряженность электрического поля, в том числе и в соседней яме QW2, вследствие кулоновской экранировки. Изменение поля приводит к изменению перекрытия волновых функций электронов и дырок и соответственно к изменению интенсивности излучения. Таким образом, на интенсивность ФЛ может влиять как процесс ближнепольного перепоглощения, так и изменение напряженности встроенного электрического поля. Проведенные эксперименты показали, что изменение интенсивности ФЛ может значительно (в 2 и более раз) превышать теоретически оцененную величину для ближнепольного эффекта, а в ряде экспериментов наблюдается и уменьшение интен- 
сивности ФЛ широкой КЯ при резонансном возбуждении экситонных переходов в узкой КЯ.

\section{4. Заключение}

Предложенная в данной работе методика измерений спектров ВФЛ позволила наблюдать экситонные линии ФЛ при непосредственном возбуждении на длине волны перехода. В результате был уточнен вклад ближнепольного взаимодействия в изменение интенсивности ФЛ. В спектрах ФЛ при изменении длины волны возбуждения наблюдались малые изменения интенсивности ФЛ и сдвиги линий, связанные с изменением встроенного электрического поля. Теоретически предсказанное в [9] увеличение интенсивности ФЛ за счет процесса ближнепольного переноса не может полностью объяснить полученные экспериментальные результаты. Возможно, это связано с необходимостью учитывать также изменение встроенного электрического поля.

Работа выполнена при поддержке РФФИ (грант № 14-02-00635) и Программы фундаментальных исследований президиума РАН.

\section{Список литературы}

[1] A. Tomita, J. Shah, R.S. Knox. Phys. Rev. B, 53, 10793 (1996).

[2] D.S. Kim, H.S. Ko, Y.S. Lim et al.. J. Opt. Soc. Am. B, 13, 1210 (1996).

[3] S.K. Lyo. Phys. Rev. B, 62, 13641 (2000).

[4] Т.С. Шамирзаев, Д.В. Дмитриев, Л.Л. Свешникова, П. Тронк. Письма ЖЭТФ, 94, 828 (2011).

[5] Th. Förster. Ann. Phys. (Leipzig), 2, 55 (1948).

[6] D.L. Dexter. J. Chem. Phys., 21, 836 (1953).

[7] В.Я. Алешкин, Л.В. Гавриленко, Д.М. Гапонова, З.Ф. Красильник, Д.И. Крыжков, Д.И. Курицын, С.М. Сергеев, В.Г. Лысенко. Письма ЖЭТФ, 94, 890 (2011).

[8] С.А. Стоклицкий, В.Н. Мурзин, Г.К. Расулова, Ю.А. Митягин, А.П. Пересторонин, Б. Монемар, О.П. Хольц, М. Сингх. Письма ЖЭТФ, 61, 399 (1995).

[9] В.Я. Алешкин, Л.В. Гавриленко, Д.М. Гапонова, Д.И. Крыжков, А.М. Кадыков, В.Г. Лысенко, З.Ф. Красильник. ЖЭТФ, 144, 1080 (2013).

[10] E.E. Mendez, F. Agulló-Rueda, J.M. Hon. Phys. Rev. Lett., 60, 2426 (1988).

Редактор Л.В. Шаронова

\section{Exiton excitation transfer processes and relaxation in low-dimentional semiconductor heterostructures with quantum wells}

\author{
V.Ya. Aleshkin ${ }^{+*}$, L.V. Gavrilenko+*, \\ D.M. Gaponova ${ }^{+*}$, Z.F. Krasil'nik ${ }^{+*}$, \\ D.I. Kryzhkov ${ }^{+*}$ \\ + Institute for Physics of Microstructures, \\ Russian Academy of Sciences, \\ 603950 Nizhny Novgorod, Russia \\ * Lobachevsky State University of Nizhny Novgorod, \\ 603950 Nizhny Novgorod, Russia
}

\begin{abstract}
The processes associated with the transfer of exciton excitations between tunnel-uncoupled quantum wells and the influence of the local electric field were investigated in $\mathrm{AlGaAs} / \mathrm{GaAs}$ heterostructures by the method of photoluminescence excitation spectroscopy at low temperature $(4.2 \mathrm{~K})$. The variation in the intensity of photoluminescence from the wider quantum well under resonance excitation of excitonic transitions in the adjacent narrow quantum well has been observed. The difference in the photoluminescence maximum position and intensity of the wider quantum well under resonance excitation of the narrow one is explained by the influence of quantum-confined Stark effect on the process of exciton recombination
\end{abstract}

